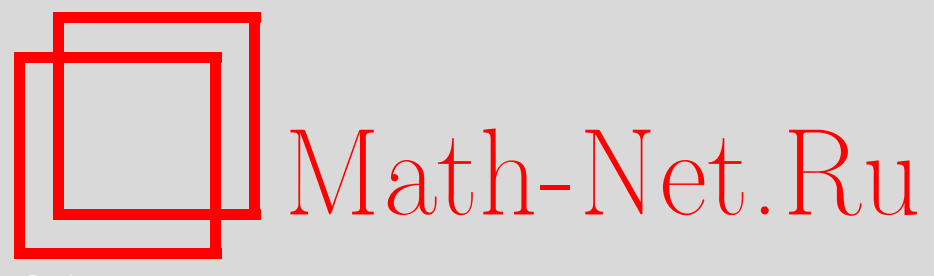

Г. Г. Гусев, Эйлерова характеристика многообразия бифуркаций для многочлена степени 2, УМH, 2008, том 63, выпуск 2, 167-168

DOI: https://doi.org/10.4213/rm9070

Использование Общероссийского математического портала Math-Net.Ru подразумевает, что вы прочитали и согласны с пользовательским соглашением http://www.mathnet.ru/rus/agreement

Параметры загрузки:

IP : 54.166 .219 .16

26 апреля 2023 г., 15:03:06

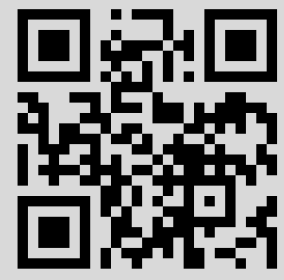




\section{Эйлерова характеристика многообразия бифуркаций для многочлена степени 2}

\section{Г. Г. Гусев}

Пусть коэффициенты многочлена $P_{\mathbf{x}}(t)=P_{0}(\mathbf{x}) t^{k}+P_{1}(\mathbf{x}) t^{k-1}+\cdots+P_{k}(\mathbf{x})$ суть многочлены Лорана от комплексных переменных $\left(x_{1}, \ldots, x_{n}\right)=\mathbf{x}$. Пространство параметров $\mathbb{C}^{* n}\left(\mathbb{C}^{*}=\mathbb{C} \backslash\{0\}\right)$ разбивается на страты, соответствующие различным комбинациям совпадения корней многочлена $P_{\mathbf{x}}$. Для многочленов $P_{0}, P_{1}, \ldots, P_{k}$ общего положения с фиксированными многогранниками Ньютона $\Delta_{0}, \Delta_{1}, \ldots, \Delta_{k}$ эйлеровы характеристики этих стратов также фиксированы. В работе получены формулы, выражающие их в терминах многогранников $\Delta_{i}$ для случая $k=2$.

При $k=2$ множество $\mathbb{C}^{* n}$ параметров разбивается на 5 стратов: 1$) K: \operatorname{deg}\left(P_{\mathbf{x}}\right)=2$, корни $P_{\mathbf{x}}$ различны; 2) $L: \operatorname{deg}\left(P_{\mathbf{x}}\right)=2$, корни совпадают; 3) $M: \operatorname{deg}\left(P_{\mathbf{x}}\right)=1$; 4) $N$ : $\operatorname{deg}\left(P_{\mathbf{x}}\right)=0$; 5) $O: P_{\mathbf{x}} \equiv 0$.

Пусть $S_{1}, \ldots, S_{n}$ - выпуклые подмножества в $\mathbb{R}^{n}$. Смешанный объем набора $S_{1}, \ldots, S_{n}$ (см. [1]) обозначим $S_{1} \cdots S_{n}$. В частности, $S_{1}^{n}$ означает $n$-мерный объем множества $S_{1}$. Для однородного многочлена $T\left(x_{1}, \ldots, x_{k}\right)=\sum \alpha_{i_{1} \cdots i_{n}} x_{i_{1}} \cdots x_{i_{n}}$ степени $n$ выражение $T\left(S_{1}, \ldots, S_{k}\right)$ будем использовать для $\sum \alpha_{i_{1} \cdots i_{n}} S_{i_{1}} \cdots S_{i_{n}}$.

Теорема 1. Для общих многочленов Лорана $P_{i}$ с данными многогранниками Нъютона $\Delta_{i}, i=0,1,2$, имеем:

$$
\begin{aligned}
\chi(L) & =(-1)^{n-1} n !\left[2 \Delta_{*}^{n}+Q_{n}\left(\Delta_{0}, \Delta_{*}\right)+Q_{n}\left(\Delta_{*}, \Delta_{2}\right)+Q_{n}\left(\Delta_{0}, \Delta_{1}\right)+R_{n}\left(\Delta_{0}, \Delta_{1}, \Delta_{2}\right)\right], \\
\chi(M) & =(-1)^{n-1} n !\left[\Delta_{0}^{n}+Q_{n}\left(\Delta_{0}, \Delta_{1}\right)\right], \\
\chi(N) & =(-1)^{n-2} n !\left[Q_{n}\left(\Delta_{0}, \Delta_{1}\right)+R_{n}\left(\Delta_{0}, \Delta_{1}, \Delta_{2}\right)\right], \\
\chi(O) & =(-1)^{n-1} n ! R_{n}\left(\Delta_{0}, \Delta_{1}, \Delta_{2}\right),
\end{aligned}
$$

где $\Delta_{*}=\left\langle\Delta_{1} \cup\left(\Delta_{0}+\Delta_{2}\right) / 2\right\rangle,\langle\cdot\rangle$ обозначает выпуклую оболочку, + - сумма Минковского, $Q_{n}(x, y):=\sum_{i=1}^{n-1} x^{i} y^{n-i}, R_{n}(x, y, z):=\sum_{i, j, k \geqslant 1, i+j+k=n} x^{i} y^{j} z^{k}$.

Пусть система многочленов Лорана от $n$ переменных $f_{1}, \ldots, f_{p}$ невырождена относительно своих многогранников Ньютона $\Delta_{1}, \ldots, \Delta_{p} ; W \subset \mathbb{C}^{* n}-$ множество нулей системы $\left\{f_{i}\right\}$. В [2] доказано, что $\chi(W)=n !\left[\prod_{i=1}^{p} \Delta_{i} /\left(1+\Delta_{i}\right)\right]_{n}$, где $[\cdot]_{n}$ - однородная часть степени $n$ рассматриваемого ряда. В частности, при $p=1,2,3$ получаем соответственно:

$$
\begin{gathered}
\chi(W)=(-1)^{n-1} n ! \Delta_{1}^{n}, \\
\chi(W)=(-1)^{n-2} n ! Q_{n}\left(\Delta_{1}, \Delta_{2}\right), \quad \chi(W)=(-1)^{n-3} n ! R_{n}\left(\Delta_{1}, \Delta_{2}, \Delta_{3}\right) .
\end{gathered}
$$

Множества $O, N \sqcup O, M \sqcup N \sqcup O$ задаются соответственно системами $\left\{P_{0}=P_{1}=\right.$ $\left.P_{2}=0\right\},\left\{P_{0}=P_{1}=0\right\},\left\{P_{0}=0\right\}$ (невырожденными для общих $P_{i}$ ). Применяя к ним формулы (1) и используя аддитивность эйлеровой характеристики, находим $\chi(M)$, $\chi(N), \chi(O)$. Следующее рассуждение, аналогичное доказательству леммы 2 из [3], позволяет найти $\chi(L)$.

Пусть $X$ - гиперповерхность в $\mathbb{C}^{* n} \times \mathbb{C}$, заданная уравнением $P=0$, где $P(\mathbf{x}, t)=$ $P_{\mathbf{x}}(t) ; p: \mathbb{C}^{* n} \times \mathbb{C} \rightarrow \mathbb{C}^{* n}$ - проекция на первый сомножитель, $\pi$ - ее ограничение на $X$. Характеристики стратов $K, L, M, N, O$ и многообразия $X$ связаны двумя линейными уравнениями. Первое уравнение $\chi(K)+\chi(L)+\chi(M)+\chi(N)+\chi(O)=0$ справедливо в силу аддитивности эйлеровой характеристики (левая часть есть эйлерова характеристика комплексного тора $\left.\mathbb{C}^{* n}\right)$. Второе уравнение - результат вычисления

Работа выполнена при поддержке РФФИ (грант № 07-01-00593) и NWO-RFBR (грант № 047.011.2004.026). 
интеграла по эйлеровой характеристике $\chi(X)=\int_{\mathbb{C}^{* n}} \chi\left(\pi^{-1}(\mathbf{x})\right) d \chi$. Прообраз точки $\mathbf{x} \in K$ состоит из двух точек, прообраз точки $\mathbf{x} \in L \sqcup M-$ из одной точки, прообраз множества $N$ пуст, и, наконец, для точки $\mathbf{x} \in O$ имеем: $\pi^{-1}(\mathbf{x}) \cong \mathbb{C}, \chi\left(\pi^{-1}(\mathbf{x})\right)=1$. Таким образом, $\chi(X)=2 \chi(K)+\chi(L)+\chi(M)+\chi(O)$. Из приведенных линейных уравнений получаем

$$
\chi(L)=-\chi(X)-\chi(M)-2 \chi(N)-\chi(O) .
$$

Найдем $\chi(X)$. Множество $X$ состоит из двух стратов: $X_{1}=X \backslash\{(\mathbf{x}, t) \mid t=0\}$ и $X_{2}=X \cap\{(\mathbf{x}, t) \mid t=0\}$. Имеем: $\chi(X)=\chi\left(X_{1}\right)+\chi\left(X_{2}\right)$. Невырожденное уравнение $P_{2}(\mathbf{x})=0$ задает множество $X_{2}$ в $\mathbb{C}^{* n}$, так что по формулам (1) находим: $\chi\left(X_{2}\right)=$ $(-1)^{n-1} n ! \Delta_{2}^{n}$. Для общих $P_{i}$ многочлен Лорана $P$, задающий в торе $\mathbb{C}^{*(n+1)}$ страт $X_{1}$, также является невырожденным, поэтому

$$
\chi\left(X_{1}\right)=(-1)^{n}(n+1) ! \Delta^{n+1},
$$

где $\Delta$ обозначает многогранник Ньютона многочлена $P$.

Выразим объем многогранника $\Delta \subset \mathbb{R}^{n+1}$ в терминах $\Delta_{i}$. Координату в пространстве $\mathbb{R}^{n+1}$, отвечающую переменной $t$, обозначим $k_{t}$. Вектор в $\mathbb{R}^{n}$ с единственной ненулевой координатой $k_{t}=1$ обозначим $v_{t}$. Многогранники $\Delta_{i}$ лежат в гиперплоскости $k_{t}=0$, многогранник $\Delta$ является выпуклой оболочкой их параллельных сдвигов вдоль оси $k_{t}: \Delta=\left\langle\left(\Delta_{0}+2 v_{t}\right) \cup\left(\Delta_{1}+v_{t}\right) \cup \Delta_{2}\right\rangle$. Нетрудно показать, что гиперплоскость $k_{t}=1$ пересекает многогранник $\Delta$ по многограннику $\Delta_{*}+v_{t}$, где $\Delta_{*}$ - многогранник, определенный в теореме 1 . Объемы частей $\left\langle\left(\Delta_{0}+2 v_{t}\right) \cup\left(\Delta_{*}+v_{t}\right)\right\rangle$ и $\left\langle\left(\Delta_{*}+v_{t}\right) \cup \Delta_{2}\right\rangle$, на которые делится многогранник $\Delta$, можно найти по следующей формуле.

Лемма 1. Пусть многогранники $\Lambda_{0}, \Lambda_{1}$ лежат в $n$-мерных гиперплоскостях, заданных в $\mathbb{R}^{n+1}$ уравнениями $x_{1}=0, x_{1}=k$ соответственно. Тогда $(n+1)$-мерный объем их выпуклой оболочки $\Lambda$ равен

$$
\Lambda^{n+1}=(n+1)^{-1} k\left(\Lambda_{0}^{n}+Q_{n}\left(\Lambda_{0}, \Lambda_{1}\right)+\Lambda_{1}^{n}\right) .
$$

ДокАзАтельство. Для $\alpha \in[0,1]$ сечение $\Lambda_{\alpha}$ многогранника $\Lambda$ гиперплоскостью $x_{1}=k \alpha$ состоит из точек вида $(1-\alpha) \mathbf{x}+\alpha \mathbf{y}$, где $\mathbf{x} \in \Lambda_{0}, \mathbf{y} \in \Lambda_{1}$. Таким образом, $\Lambda_{\alpha}=$ $(1-\alpha) \Lambda_{0}+\alpha \Lambda_{1}$ и, значит, $\Lambda^{n+1}=k \int_{[0,1]}\left(\Lambda_{\alpha}\right)^{n} d \alpha=k \int_{[0,1]}\left[\sum_{i=0}^{n} C_{n}^{i} \alpha^{i}(1-\alpha)^{n-i}\left(\Lambda_{1}\right)^{i} \times\right.$ $\left.\left(\Lambda_{0}\right)^{n-i} d \alpha\right]$. Учитывая, что $\int_{[0,1]}\left(\alpha^{i}(1-\alpha)^{n-i}\right) d \alpha=i !(n-i) ! /(n+1) !=1 /\left((n+1) C_{n}^{i}\right)$, получаем формулу (4).

Применяя (4) к частям многогранника $\Delta$ и пользуясь инвариантностью смешанных объемов относительно параллельного переноса аргументов, получаем: $\Delta^{n+1}=$ $(n+1)^{-1}\left(\Delta_{0}^{n}+2 \Delta_{*}^{n}+\Delta_{2}^{n}+Q_{n}\left(\Delta_{0}, \Delta_{*}\right)+Q_{n}\left(\Delta_{*}, \Delta_{2}\right)\right)$. Теперь формула теоремы 1 для $\chi(L)$ следует из $(2),(3)$.

Автор благодарен С.М. Гусейн-Заде за постановку задачи и содействие в ее решении.

\section{Список литературы}

[1] Г. Буземан, Выпуклъе поверхности, Наука, М., 1964. [2] А. Г. Хованский, Функц. анализ и его прил., 12:1 (1978), 51-61. [3] А. И. Эстеров, Матем. сб., 197:7 (2006), 137-160.

\section{Г. Г. Гусев (G. G. Gusev)}

Московский государственный университет им. М. В. Ломоносова

E-mail: gusev@mccme.ru
Представлено С. М. Гусейн-Заде Принято редколлегией 17.12.2007 